\title{
Biased Positional Games and Small Hypergraphs with Large Covers
}

\author{
Michael Krivelevich * Tibor Szabó ${ }^{\dagger}$ \\ Submitted: Feb 24, 2007; Accepted: May 1, 2008; Published: May 5, 2008 \\ Mathematics Subject Classification: 91A43, 91A46, 05C65
}

\begin{abstract}
We prove that in the biased $(1: b)$ Hamiltonicity and $k$-connectivity MakerBreaker games $(k>0$ is a constant), played on the edges of the complete graph $K_{n}$, Maker has a winning strategy for $b \leq(\log 2-o(1)) n / \log n$. Also, in the biased $(1: b)$ Avoider-Enforcer game played on $E\left(K_{n}\right)$, Enforcer can force Avoider to create a Hamilton cycle when $b \leq(1-o(1)) n / \log n$. These results are proved using a new approach, relying on the existence of hypergraphs with few edges and large covering number.
\end{abstract}

\section{Introduction}

Positional games involve two players who alternately occupy the elements of a given set $V$, the board of the game. The focus of their attention is a given family $\mathcal{F}=\left\{e_{1}, \ldots, e_{k}\right\} \subseteq 2^{V}$ of subsets of $V$, usually called the family of winning sets. The players exchange turns occupying one previously unoccupied element of $V$. The game ends when there are no unoccupied elements of $V$. The game is specified completely by defining who wins in every final position or, more generally, for every possible game scenario. An interested reader is invited to read several survey papers of József Beck on the subject (see, e.g., [4]), and also his forthcoming book [5] for an extensive background on this fascinating subject.

There are several types of positional games depending on how the identity of the winner is determined. In this paper we restrict our attention to two of them.

\footnotetext{
${ }^{*}$ School of Mathematical Sciences, Raymond and Beverly Sackler Faculty of Exact Sciences, Tel Aviv University, Tel Aviv, 69978, Israel. Email: krivelev@post.tau.ac.il. Research supported in part by a USA-Israel BSF grant, by a grant from the Israel Science Foundation and by the Pazy Memorial Award.

†Institute of Theoretical Computer Science, ETH Zurich, CH-8092 Switzerland. Email: szabo@inf.ethz.ch.
} 


\subsection{Maker-Breaker games}

In a Maker-Breaker game the first player, called Maker, wins if he completely occupies one of the winning sets by the end of the game; the second player, called Breaker, wins otherwise, i.e., if he manages to occupy at least one element of (i.e., "to break into") every winning set by the end of the game.

Chvátal and Erdős [8] were the first to explicitly consider Maker-Breaker positional games which are played on the edge set of the complete graph $K_{n}$, and the winning sets are defined by some graph theoretic property like connectivity or Hamiltonicity. In the connectivity game Maker wins if he creates a spanning tree by the end of the game. In the Hamiltonicity game Maker wins if his graph contains a Hamilton cycle in the end.

It seems that Maker, partly because he has so many (i.e., $\frac{1}{2}\left(\begin{array}{l}n \\ 2\end{array}\right)$ ) edges by the end, should be able to win both of these games easily. This is indeed the case; for the connectivity game the winning strategy is a triviality, for the Hamiltonicity game it requires a one-page argument [8]. Motivated by the easy success of Maker, Chvátal and Erdős suggested to make the game more balanced by introducing a bias: at each turn Breaker is allowed to occupy $b$ edges instead of just one, where $b \geq 1$ is an integer. In these games, as well as in other $(1: b)$ games, the most natural question is, for a given value of the bias $b$, to determine which of the two players wins, assuming of course they play their optimal strategies. Chvátal and Erdős proved [8] that if $b<\left(\frac{1}{4}-o(1)\right) \frac{n}{\log n}$ then Maker can still occupy a spanning tree and thus win the connectivity game. They also proved [8] that the order of magnitude of the bias is best possible. In fact they showed that if $b>(1+o(1)) \frac{n}{\log n}$ then Breaker can occupy all edges incident to some vertex, so Maker loses the connectivity game, since his graph is disconnected. Later Beck [1] improved the constant factor in the result of Chvátal and Erdős and established that Maker wins the connectivity game even if $b<(\log 2-o(1)) \frac{n}{\log n}$.

For the Hamiltonicity game Chvátal and Erdős conjectured that there is function $b_{\mathcal{H}}(n)$ tending to infinity such that Maker can still build a Hamilton cycle if he plays against a bias $b_{\mathcal{H}}(n)$. Their conjecture was verified by Bollobás and Papaioannou [7] who proved that Maker is able to build a Hamilton cycle even if Breaker's bias is as large as $\frac{c \log n}{\log \log n}$. Beck improved greatly on this [2] and established that the order of magnitude of the critical bias is the same for the Hamiltonicity game and the connectivity game. He showed that Maker wins the Hamiltonicity game provided Breaker's bias is at most $\left(\frac{\log 2}{27}-o(1)\right) \frac{n}{\log n}$.

In this paper we improve the constant factor in the Hamiltonicity game result of Beck and achieve the same lower bound as is known for the connectivity game.

Theorem 1 Maker wins the $(1: b)$ Hamiltonicity game for every $b<(\log 2-o(1)) \frac{n}{\log n}$.

Our proof technique provides the same lower bound for the critical bias in the $k$ connectivity game, where the family $\mathcal{C}_{k}$ of winning sets consists of the edgesets of $k$ connected spanning graphs. 
Theorem 2 Maker wins the $(1: b) k$-connectivity game for every $b<(\log 2-o(1)) \frac{n}{\log n}$.

\subsection{Avoider-Enforcer games}

We also study the misère version of Maker-Breaker games. In an Avoider-Enforcer game the first player, called Avoider, wins if he does not occupy completely any of the winning sets by the end of the game; otherwise the second player, called Enforcer, wins.

Although Avoider-Enforcer games appear naturally in several situation related to Maker-Breaker games, our understanding of them is much less satisfactory. The AvoiderEnforcer versions of graph games often behave surprising differently from their MakerBreaker counterparts. For example Hefetz and the authors [13] recently proved that in the $(1: b)$ Avoider-Enforcer connectivity game the critical bias is linear in $n$ : Avoider wins if and only if the bias of Enforcer is at least $(1+o(1)) n / 2$. This is in striking contrast with the order $\frac{n}{\log n}$ of the critical bias in the Maker-Breaker connectivity game. The AvoiderEnforcer Hamiltonicity game was also analyzed in [13], where it was proven that if the bias of Enforcer is $O\left(\frac{n \log ^{(4)} n}{\log n \log ^{(3)} n}\right)$ then Enforcer wins (the notation $\log ^{(k)} n$ stands for the $k$ times iterated logarithm of $n$ ). Here we improve this result and obtain the same order of magnitude as for the Maker-Breaker games — with a better constant. The theorem resolves one of the "seven most humiliating open problems" listed in the book of Beck [5].

Theorem 3 Enforcer wins the $(1: b)$ Hamiltonicity game for every $b<(1-o(1)) \frac{n}{\log n}$.

A major difference we have here compared to the Maker-Breaker counterpart is the complete lack of results from the other direction. While we know the order of magnitude of the critical bias in the Maker-Breaker Hamiltonicity game, for Avoider's win the best available strategy is the trivial one: Avoider (clearly) wins if he has less then $n$ edges by the end of the game (which happens for bias $b=(1+o(1)) n / 2)$.

Similarly to Maker-Breaker games, our technique carries the bound through to $k$ connectivity games.

Theorem 4 Enforcer wins the $(1: b) k$-connectivity game for every $b<(1-o(1)) \frac{n}{\log n}$.

To the best of our knowledge the $k$-connectivity game (in both the Maker-Breaker and Avoider-Enforcer versions) has not been considered explicitly in the literature for $k \geq 2$.

\subsection{Notation and Terminology}

A hypergraph $\mathcal{F}$ is a subset of the power set $2^{V}$ where $V$ is a finite non-empty set (vertex set). The members of $\mathrm{F}$ are called hyperedges. We say that a positional game is played on a hypergraph $\mathcal{F}$ where $V$ is the board of the game, and $\mathcal{F}$ is the collection of winning sets. The identity of the player making the first move does not affect our asymptotic results; unless otherwise stated, we set as a default that Maker or Avoider starts the games. If all hyperedges of a hypergraph $\mathcal{F}$ are of size $r$, then $\mathcal{F}$ is called $r$-uniform. A subset $T \subseteq V$ 
is a cover of $\mathcal{F}$ if $T \cap e \neq \emptyset$ for every $e \in \mathcal{F}$. The covering number of $\mathcal{F}$, denoted by $\tau(\mathcal{F})$, is the minimal cardinality of a cover of $\mathcal{F}$.

For a graph $G$ and a subset $U \subset V(G)$, we denote by $N(U)$ the external neighborhood of $U$, i.e., $N(U)=\{v \in V(G) \backslash U: v$ has a neighbor in $U\}$.

Whenever needed we assume that the underlying parameter $n$ is large enough. All logarithms are natural unless stated explicitly otherwise. We will ignore systematically rounding and divisibilty issues.

\section{The random graph intuition and open problems}

In this section we discuss a paradigm, pointed out first by Chvátal and Erdős [8] and covered later in great detail in several papers of Beck (c.f. [3]). This paradigm relates positional games to random graphs. The goal of the section is twofold. On the one hand it describes this paradigm, called "random graph intuition", which is a major driving force behind our current results. On the other hand, we list here some of the open problems we find interesting which are motivated by the random graph intuition.

Biased positional games, as defined above, are perfect information games played by two players, and as such they apparently do not leave much room for probabilistic considerations. Yet, as the accumulated research experience convincingly shows, probabilistic intuition is of utmost importance when analyzing biased games. For biased $(1: b)$ games, the most important question is who wins for a given value of bias $b$. It is often quite easy to see that for small enough $b$ Maker wins, while when $b$ is large enough the win is Breaker's. It is therefore natural to ask for the critical value of $b$ at which the game "changes hands" and becomes Breaker's win instead of Maker's One can easily prove formally that there is bias monotonicity, i.e., the game changes hands at most once. More formally, for a given hypergraph $\mathcal{F}$ let $b_{\mathcal{F}}$ be the largest integer $b$ for which Maker wins the $(1: b)$ game played on the hypergraph $\mathcal{F}$. The integer $b_{\mathcal{F}}$ is called the critical bias of the positional game $\mathcal{F}$. It can easily be proved formally that Maker wins the $(1: b)$ game for every $b \leq b_{\mathcal{F}}$.

Paul Erdős suggested the following heuristic argument to guess the critical bias. During the game Maker claims $m \approx \frac{n^{2}}{2(b+1)}$ edges, the rest belongs to Breaker. Of course, both players are assumed to play perfectly. Yet, if instead each of them would play completely at random, RandomMaker's graph would look like a random graph with $n$ vertices and $m$ edges. This model of random graphs is usually denoted by $G(n, m)$. Erdös suggested to look at the critical $m$ for which the corresponding combinatorial property (connectivity, Hamiltonicity etc.) starts appearing almost surely in $G(n, m)$ as a hint for the critical bias $b$. Since the theory of random graphs is a rather well developed field, where quite a few questions about the location of a threshold for the appearance of various combinatorial properties have been solved successfully, Erdős' paradigm allows for guessing the critical bias for a variety of biased positional games. And indeed, for the connectivity and Hamiltonicity games the results obtained fit Erdős' prediction surprisingly well. In 
order to convince the reader in this thesis, and also as a future reference point, we state known results about connectivity and Hamiltonicity in $G(n, m)$. It is well known that, for any constant $k \geq 1$, if $m=m(n)=\frac{n}{2}(\log n+(k-1) \log \log n-\omega(n))$, then the random graph $G(n, m)$ has almost surely a vertex of degree at most $k-1$ and is thus not $k$-connected. On the other hand, if $m=m(n)=\frac{n}{2}(\log n+(k-1) \log \log n+\omega(n))$, then $G(n, m)$ is almost surely $k$-connected [9]. (The term "almost surely" means "with probability tending to 1 as the number of vertices $n$ tends to infinity"; $\omega(n)$ stands for any function tending to infinity abritrarily slowly with $n$.) As for Hamiltonicity, applying the above lower bound for $k=2$, one can derive that $G(n, m)$ is almost surely nonHamiltonian for $m=m(n)=\frac{n}{2}(\log n+\log \log n-\omega(n))$. Komlós and Szemerédi [15] and independently Bollobás [6] proved that if $m=m(n)=\frac{n}{2}(\log n+\log \log n+\omega(n))$ then $G(n, m)$ is almost surely Hamiltonian. The above statements suggest that the critical bias $b$ for both the connectivity and Hamiltonicity games should be of order $n / \log n$, and this is indeed matched by available results on positional games. (One should note though that upon a closer inspection the positional games results are sizably less accurate than their random graphs' counterparts.)

The results of the current paper unify the known lower bounds for all the above games. Denote by $b_{\mathcal{D}_{k}}, b_{\mathcal{C}_{k}}, b_{\mathcal{H}}, b_{\mathcal{T}}$ the critical biases for the minimum degree $k, k$-connectivity, Hamiltonicity and connectivity games, respectively. We now have that

$$
(\log 2-o(1)) \frac{n}{\log n} \leq b_{\mathcal{H}} \leq b_{\mathcal{T}} \leq b_{\mathcal{D}_{1}} \leq(1+o(1)) \frac{n}{\log n}
$$

and

$$
(\log 2-o(1)) \frac{n}{\log n} \leq b_{\mathcal{C}_{k}} \leq b_{\mathcal{D}_{k}} \leq b_{\mathcal{D}_{1}} \leq(1+o(1)) \frac{n}{\log n} .
$$

Hence the foremost obstacle standing in the way of the asymptotic determination of the critical bias for the connectivity or Hamiltonicity game is the inability of our current techniques to deal with the mindegree-1 game. In other words, what is the smallest bias of Breaker which allows him to isolate a vertex in Maker's graph?

Problem 1 Determine $b_{\mathcal{D}_{1}}$ asymptotically.

In the model of random graph process an even stronger connection is true between, say, the properties of connectivity and mindegree-1. In an informal language this says that the main reason a random graph is not connected is that there exists an isolated vertex. This motivates our question whether a similar phenomenon holds in the theory of biased positional games. We think the answer is yes, i.e., the only reason Maker cannot win the connectivity game is that Breaker is able to isolate a vertex in Maker's graph.

\section{Conjecture 1}

$$
b_{\mathcal{T}}=b_{\mathcal{D}_{1}}
$$

We are curious whether one can show anything of this sort without obtaining the asymptotic value of these critical biases. Other natural questions motivated by known facts on random graph processes are 
- Is it true that $b_{\mathcal{H}}=b_{\mathcal{D}_{2}}$ ?

- Is it true that $b_{\mathcal{C}_{k}}=b_{\mathcal{D}_{k}}$ ?

For Avoider-Enforcer games our knowledge of the critical bias is much more limited than for the Maker-Breaker case. In fact the situation is more complicated as AvoiderEnforcer games lack bias-monotonicity in general [13]. Still, at least for some of these games the above described Erdős paradigm can serve as a very good guess in deciding the winner for a given value of bias $b$. We would like to close the open problem section with an admittedly modest conjecture which just highlights our lack of understanding of Avoider-Enforcer games.

Conjecture 2 Prove that Avoider has a winning strategy in the (1: $\left.\frac{n}{10}\right)$ Hamiltonicity game.

\section{Tools of the trade}

For our proofs one needs to extend the concept of positional games so that Maker and Avoider can also have a bias larger than one. Given a hypergraph $\mathcal{F}$ on a vertex set $V$, in a $(p: q)$ positional game the first player occupies $p$ element of $V$ in each round, while the second player occupies $q$ in each round.

One of the most important technical tools in the theory of biased positional games is the following criterion for Breaker's win due to Beck [1]. If

$$
\sum_{e \in \mathcal{F}}(1+q)^{-|e| / p}<\frac{1}{1+q}
$$

then Breaker has a winning strategy (irregardless of who moves first). Historically, since Erdős and Selfridge established the above criterion for the unbiased case $p=q=1$ in [10], inequality (1) is often called the biased Erdős-Selfridge criterion.

The Avoider-Enforcer version of the above criterion has been obtained recently in [13] (see also [5]). In a $(p: q)$ biased Avoider-Enforcer game, played on a collection $\mathcal{F}$ of winning sets, Avoider wins provided

$$
\sum_{e \in \mathcal{F}}\left(1+\frac{1}{p}\right)^{-|e|}<\left(1+\frac{1}{p}\right)^{-p} .
$$

One should note that the above criterion does not involve the parameter $q$ at all and is thus less powerful compared to the corresponding Maker-Breaker condition (1) of Beck. Nevertheless, the latter criterion will be good enough for our purposes. 


\section{An example}

In this section we present and discuss a relatively simple example, whose purpose is to illustrate a standard approach to analyzing biased positional games, its limitations, and also to indicate the main idea behind our proof.

Consider the following $(1: b)$ Maker-Breaker game played on the edges of the complete graph $K_{n}$. An additional parameter $k \geq 1$ is given (the reader should think about $k$ as a constant). Maker wins the mindegree- $k$ game if his graph has minimum degree at least $k$ by the end. The corresponding hypergraph, whose members are the edgesets of graphs with minimum degree $k$, is denoted by $\mathcal{D}_{k}$. Observe that according to the above cited Chvátal-Erdös result [8] when $b \geq(1+o(1)) n / \log n$, Breaker can isolate a vertex and thus win the mindegree- $k$ game for every positive $k$. We will be interested in finding the bias $b$ as large as possible for which Maker still wins, and therefore in finding good strategies and criteria for Maker's win in the mindegree- $k$ game.

As is the case with quite a few combinatorial games, in order for Maker to achieve a win, it turns out very useful to interpret the game differently and to view Maker as playing Breaker's role in this different interpretation. This approach was first suggested by Beck in [1] and later used frequently by him and other authors. Its main advantage is in the possibility of applying criterion (1) for Breaker's win in biased games.

For each $v \in V\left(K_{n}\right)$, define a hypergraph $\mathcal{F}_{v}$ composed of all sets of $n-k$ edges of $K_{n}$ incident to $v$. Clearly, $\mathcal{F}_{v}$ is an $(n-k)$-uniform hypergraph with $\left|E\left(\mathcal{F}_{v}\right)\right|=\left(\begin{array}{l}n-1 \\ n-k\end{array}\right)=\left(\begin{array}{l}n-1 \\ k-1\end{array}\right)$ hyperedges. Observe that if Maker manages to occupy an edge (of $K_{n}$ ) in every hyperedge of $\mathcal{F}_{v}$, then he has at least $k$ edges incident to $v$. (If this is not the case, then Breaker has some $n-1-(k-1)=n-k$ edges incident to $v$ in his possession, those edges form a hyperedge of $\mathcal{F}_{v}$ completely occupied by Breaker.) Therefore, in order for Maker to win the mindegree- $k$ game, it suffices to break into every hyperedge of the hypergraph $\mathcal{F}=\bigcup_{v \in V\left(K_{n}\right)} \mathcal{F}_{v}$. The hypergraph $\mathcal{F}$ is $(n-k)$-uniform with $n\left(\begin{array}{l}n-1 \\ k-1\end{array}\right)$ edges. Applying the biased Erdős-Selfridge criterion (1) to $\mathcal{F}$ and substituting $p=b, q=1$, it follows that if

$$
n \cdot\left(\begin{array}{l}
n-1 \\
k-1
\end{array}\right) 2^{-\frac{n-k}{b}}<\frac{1}{2}
$$

then Maker wins the mindegree- $k$ game. Standard asymptotic manipulations show that the largest $b=b(n)$ for which the above inequality is valid is

$$
b=\left(\frac{\log 2}{k}+o(1)\right) \frac{n}{\log n} .
$$

Let us evaluate the obtained result. First of all, it is not that bad - the lower bound for the critical bias we have just obtained is of the same order of magnitude as the Chvátal-

Erdős upper bound and the constant factor depends only linearly on $k$. However, recalling the intuition coming from random graphs such a result is not satisfactory. Indeed, the critical number of edges $m$, for which the random graph $G(n, m)$ starts having almost 
surely minimum degree at least $k$, depends on $k$ very weakly - it is $\left(\frac{1}{2}+o(1)\right) n \log n$ for any constant $k$.

Let us return to our argument. A simple observation, playing a crucial role in the current paper, is that the most important property of the hypergraph $\mathcal{F}_{v}$ was the fact that its covering number is at least $k$ (actually, it is exactly $k$ ). If Maker hits every hyperedge of $\mathcal{F}_{v}$ during the game then his edges incident to $v$ form a cover of $\mathcal{F}_{v}$, and, as $\tau\left(\mathcal{F}_{v}\right) \geq k$, we can conclude that Maker occupies at least $k$ edges of $K_{n}$ incident to $v$.

We can conclude that instead of $\mathcal{F}_{v}$ it is potentially beneficial to choose a hypergraph with fewer hyperedges of still relatively large size (to make it easier for Maker to hit each of them), yet with covering number large enough. Turning this paradigm into a quantitative relation between the parameters involved will play a key role in deriving the improved results. We are thus interested in showing the existence of hypergraphs with few hyperedges and large covering number. This is discussed in the next section.

\section{$5 \quad$ Large covers of small hypergraphs}

For positive integers $n, r, t$ satisfying $r \leq n$, and $t \leq n-r+1$, define

$$
f(n, r, t)=\min \{|\mathcal{F}|: \mathcal{F} \text { is an } r \text {-uniform hypergraph on } n \text { vertices, } \tau(\mathcal{F}) \geq t\} .
$$

Claim 1 For every $s \geq t$,

$$
f\left(n, \frac{s-t+1}{s} n, t\right) \leq\left(\begin{array}{c}
s \\
t-1
\end{array}\right) .
$$

Proof. Partition $[n]=\bigcup_{i=1}^{s} A_{i},\left|A_{i}\right|=n / s$. Define a hypergraph whose vertex set is $V=[n]$, and whose hyperedge set is

$$
\mathcal{F}=\left\{\cup_{i \in I} A_{i}: I \subseteq[s],|I|=s-t+1\right\}
$$

Clearly $\mathcal{F}$ is an $\frac{s-t+1}{s} n$-uniform hypergraph. If a set $T \subset[n]$ intersects less than $t$ of the sets $A_{i}$, then there is at least one hyperedge of $\mathcal{F}$ left completely outside $T$, and thus $T$ is not a cover of $\mathcal{F}$. Therefore, $\tau(\mathcal{F}) \geq t$.

For comparison, here is a lower bound for $f(n, r, t)$. If $\mathcal{F}$ is an $r$-uniform hypergraph on $n$ vertices with $\tau(\mathcal{F}) \geq t$, then every set $U \subset V,|U|=t-1$, misses at least one member of $\mathcal{F}$, implying:

$$
|\mathcal{F}| \geq \frac{\left(\begin{array}{c}
n \\
t-1
\end{array}\right)}{\left(\begin{array}{c}
n-r \\
t-1
\end{array}\right)}
$$

In case $r=\frac{s-t+1}{s} n$ and $s, t=o(n)$, both lower and upper bounds on $f(n, r, t)$ are of the form $(c s /(t-1))^{t-1}$. 


\section{Biased Hamiltonicity game}

In this section we obtain a new estimate for the critical bias in the Maker-Breaker Hamiltonicity game. This estimate improves substantially the currently best known result due to Beck [2] and comes quite close to the upper bound of Chvátal and Erdős [8]. The proof of Theorem 1 is a combination of our basic "thinning" trick and the following Hamiltonicity criterion derived recently by Hefetz and the authors [14].

Lemma 1 Let $12 \leq d \leq e^{\sqrt[3]{\log n}}$ and let $G$ be a graph on $n$ vertices satisfying properties P1, P2 below:

$\mathbf{P 1}$ For every $S \subset V$, if $|S| \leq k_{1}(n, d):=\frac{n \log \log n \log d}{d \log n \log \log \log n}$ then $|N(S)| \geq d|S|$;

P2 There is an edge in $G$ between any two disjoint subsets $A, B \subseteq V$ such that

$$
|A|,|B| \geq k_{2}(n, d):=\frac{n \log \log n \log d}{4130 \log n \log \log \log n} .
$$

Then $G$ is Hamiltonian, for sufficiently large $n$.

In fact, conditions $\mathbf{P} \mathbf{1}$ and $\mathbf{P} 2$ above will be quite handy in the $k$-connectivity game as well. Theorem 1 is now an immediate corollary of Lemma 1 and the following theorem.

Theorem 5 Let $b=\frac{n}{\log _{2} n+\frac{\log n}{\log \log \log n}}$. In a biased $(1: b)$ Maker-Breaker game played on the edges of the complete graph $K_{n}$, Maker has a strategy to build a graph which satisfies properties $\mathbf{P} 1$ and $\mathbf{P} 2$ with $d=d(n)=\frac{\log n}{\log \log n}$, provided $n$ is large enough.

Remark. No real attempt is made here to optimize the error term in the above expression for $b$.

Proof. We introduce some notation:

$$
\begin{aligned}
& k_{1}^{*}=k_{1}^{*}(n)=k_{1}(n, d)=(1+o(1)) \frac{n(\log \log n)^{3}}{\log ^{2} n \log \log \log n}, \\
& k_{2}^{*}=k_{2}^{*}(n)=k_{2}(n, d)=(1+o(1)) \frac{n(\log \log n)^{2}}{4130 \log n \log \log \log n} .
\end{aligned}
$$

For every $1 \leq k \leq k_{1}^{*}$ and for every set $S \subset[n],|S|=k$, let $\mathcal{F}(S)$ be an $r(k)$-uniform hypergraph on $[n] \backslash S$ with at most $\left(\begin{array}{c}s(k) \\ d k\end{array}\right)$ hyperedges whose covering number satisfies: $\tau(\mathcal{F}(S)) \geq d k+1$, where

$$
s(k)=k \log n, \quad r(k)=\frac{\log n-d}{\log n}(n-k)=\left(1-\frac{1}{\log \log n}\right)(n-k) .
$$

The existence of such a hypergraph is guaranteed by Claim 1. Observe that if, by the end of the game, in Maker's graph $M$ the set $S$ is connected by an edge to every member of $\mathcal{F}(S)$, then $\left|N_{M}(S)\right| \geq \tau(\mathcal{F}(S))>d k$. Indeed, in this case the external neighborhood of $S$ in $M$ forms a cover of $\mathcal{F}(S)$. 
It thus follows that in order to ensure Condition $\mathbf{P 1}$, it is enough to guarantee that in Maker's graph $M$, for every $1 \leq k \leq k_{1}^{*}$ and for every set $S \subset[n],|S|=k, S$ is connected to every edge from $\mathcal{F}(S)$. In order to meet condition $\mathbf{P} 2$, it is enough to guarantee that in $M$ there is an edge between every pair of disjoint sets $A, B \subset[n]$ of size $|A|=|B|=k_{2}^{*}$. By the generalized Erdős-Selfridge condition (1) it is therefore enough to check that:

$$
\sum_{k=1}^{k_{1}^{*}} \sum_{|S|=k}|\mathcal{F}(S)| 2^{-\frac{k \cdot r(k)}{b}}+\sum_{|A|,|B|=k_{2}^{*}} 2^{-\frac{\left(k_{2}^{*}\right)^{2}}{b}}<\frac{1}{2},
$$

or

$$
\sum_{k=1}^{k_{1}^{*}}\left(\begin{array}{l}
n \\
k
\end{array}\right)\left(\begin{array}{c}
k \log n \\
d k
\end{array}\right) 2^{-\frac{k\left(1-\frac{1}{\log \log n}\right)(n-k)}{b}}+\left(\begin{array}{c}
n \\
k_{2}^{*}
\end{array}\right)\left(\begin{array}{c}
n-k_{2}^{*} \\
k_{2}^{*}
\end{array}\right) 2^{-\frac{\left(k_{2}^{*}\right)^{2}}{b}}<\frac{1}{2} .
$$

We will prove that both summands in the left hand side of the inequality above are asymptotically negligible. The first one can be estimated from above by:

$$
\begin{aligned}
& \sum_{k=1}^{k_{1}^{*}}\left[n\left(\frac{e \log n}{d}\right)^{d} 2^{-\left(1-\frac{1}{\log \log n}\right)\left(1-\frac{k}{n}\right)\left(\log _{2} n+\frac{\log n}{\log \log \log n}\right)}\right]^{k} \\
& \leq \sum_{k=1}^{k_{1}^{*}}\left[2^{\left.\log _{2} n+\frac{\log n}{\log \log n} \log 2(e \log \log n)-\left(1-\frac{1}{\log \log n}-\frac{k_{1}^{*}}{n}\right) \log _{2} n-\frac{1}{2} \cdot \frac{\log n}{\log \log \log n}\right]^{k}}\right. \\
& =\sum_{k=1}^{k_{1}^{*}}\left[2^{O\left(\frac{\log n \log \log \log n}{\log \log n}\right)-\frac{\log n}{2 \log \log \log n}}\right]^{k}=o(1) .
\end{aligned}
$$

The second summand is at most:

$$
\begin{aligned}
{\left[\left(\frac{e n}{k_{2}^{*}}\right)^{2} 2^{-\frac{k_{2}^{*}}{b}}\right]^{k_{2}^{*}} } & =\left[\left(\frac{O(1) \log n \log \log \log n}{(\log \log n)^{2}}\right)^{2} 2^{-\frac{\Theta(1)(\log \log n)^{2}}{\log \log \log n}}\right]^{k_{2}^{*}} \\
& \leq\left(2^{O(1) \log \log n-(\log \log n)^{1.9}}\right)^{k_{2}^{*}}=o(1) .
\end{aligned}
$$

This finishes the proof of Theorem 5 .

\section{$7 \quad$ Biased $k$-connectivity game}

In this section we prove Theorem 2. To this end we show that if the bias $b$ satisfies $b=(\log 2+o(1)) \frac{n}{\log n}$ then Maker can build a $k$-connected graph in the biased $(1: b)$ game, for any constant $k>0$ (and in fact for some slowly growing $k$ as well). Observe that this weak dependence of the obtained result on $k$ matches very well our intuition derived from random graphs and discussed in Section 2. 
Theorem 6 In a biased (1:b) Maker-Breaker game, Maker has a strategy for creating a $\frac{\log n}{\log \log n}$-connected graph provided

$$
b=\frac{n}{\log _{2} n+\frac{\log n}{\log \log \log n}},
$$

and $n$ is large enough.

Remark. Here, too, we do not make any serious attempt to get the best possible connectivity result, our aim is rather to get $k$-connectivity for some $k \gg 1$, when the bias is $b=(1-o(1)) n / \log _{2} n$.

Proof. In Theorem 5 we showed that Maker can create a graph $M$ satisfying conditions $\mathbf{P} 1$ and $\mathbf{P} 2$ of Lemma 1 with $d(n)=\frac{\log n}{\log \log n}$. We claim that every graph $M$ meeting these conditions with the above value of $d(n)$ is $d(n)$-connected. Let $S$ be a subset of $V(M)$ of cardinality $|S|<d(n)$. Assume to the contrary that $S$ cuts $G$ into two non-empty pieces: $V(M) \backslash S=A \cup B$ so that $|A| \leq|B|$ and $M$ has no edges between $A$ and $B$. We will use the notation of the proof of Theorem 5. First, observe that $|A| \geq k_{1}^{*} d-d$. Indeed, if $|A| \leq k_{1}^{*}$, then by $\mathbf{P 1}$ we have: $|N(A)| \geq d|A| \geq d$ and $N(A) \subseteq S-$ a contradiction. If $k_{1}^{*} \leq|A| \leq k_{1}^{*} d-d$, choose $A_{0} \subseteq A$ of cardinality $\left|A_{0}\right|=k_{1}^{*}$, then by $\mathbf{P} \mathbf{1}$ we have $\left|N\left(A_{0}\right)\right| \geq d\left|A_{0}\right|=k_{1}^{*} d$ and $N\left(A_{0}\right) \subseteq\left(A \backslash A_{0}\right) \cup S$, implying a claimed bound on $|A|$. But then $|A| \geq k_{2}^{*}$ and since $|B| \geq|A| \geq k_{2}^{*}$, by Property P2 there is an edge of $M$ between $A$ and $B-$ a contradiction.

Theorem 2 implies immediately the following consequence for the mindegree- $k$ game considered in Section 4.

Corollary 1 For every fixed $k>0$ Makers wins the $(1: b)$ mindegree- $k$ game provided

$$
b \leq \frac{n}{\log _{2} n+\frac{\log n}{\log \log \log n}},
$$

and $n$ is sufficiently large.

\section{Biased Avoider-Enforcer games}

For biased Avoider-Enforcer games we can use a very similar proof strategy to that of Theorem 5 to infer the following.

Theorem 7 Let $b \leq \frac{n}{\log n+\frac{\log n}{\log \log \log n}}$. In a biased $(1: b)$ Avoider-Enforcer game, Enforcer has a strategy to force Avoider to create a graph satisfying $P 1$ and and P2 with $d=d(n)=$ $\frac{\log n}{\log \log n}$ provided $n$ is large enough. 
The only major difference is that instead of the generalized Erdős-Selfridge criterion (1), Enforcer uses criterion (2) to make sure that Avoider's graph satisfies properties P1 and P2. Plugging into Criterion (2), inequality (3) now becomes

$$
\sum_{k=1}^{k_{1}^{*}} \sum_{|S|=k}|\mathcal{F}(S)|\left(1+\frac{1}{b}\right)^{-k \cdot r(k)}+\sum_{|A|,|B|=k_{2}^{*}}\left(1+\frac{1}{b}\right)^{-\left(k_{2}^{*}\right)^{2}}<\left(1+\frac{1}{b}\right)^{-b} .
$$

We can use now the asymptotic estimate $1+x=e^{x+\Theta\left(x^{2}\right)}$ for $x \rightarrow 0$. The rest of the proof remains the same mutatis mutandis.

To derive Theorem 3 we can apply Lemma 1 . For Theorem 4 we only note that, as proved in the previous section, properties $\mathbf{P} 1$ and $\mathbf{P} 2$ for $d(n)=\log n / \log \log n$ imply $d(n)$-connectivity.

Note added in proof. Very recently, Gebauer and the second author proved [11] that the thresholds bias for both the connectivity game and the mindegree- $k$ game are asymptotically equal to $n / \log n$. Also, Hefetz, Stojaković and the authors defined in [12] a new and rather natural version of the Avoider-Enforcer game and proved that in this version the threshold bias for the Hamiltonicity game equals asymptotically to $n / \log n$, where the lower bound essentially comes from the present paper.

\section{References}

[1] J. Beck, Remarks on positional games, Acta Math. Acad. Sci. Hungar. 40 (1982), $65-71$.

[2] J. Beck, Random graphs and positional games on the complete graph, Annals of Discrete Math. 28 (1985) 7-13.

[3] J. Beck, Deterministic graph games and a probabilistic intuition, Combin., Prob. Computing 3 (1994), 13-26.

[4] J. Beck, Positional games, Combin., Prob. Computing 14 (2005), 649-696.

[5] J. Beck, Combinatorial Games, Cambridge Univ. Press, 2008.

[6] B. Bollobás, The evolution of random graphs, in Graph Theory and Combinatorics (Cambridge, 1983), Academic Press, London, 1984, pp. 35-57.

[7] B. Bollobás and A. Papaioannou, A biased Hamiltonian game, Congressus Numerantium, 35 (1982), 105-115.

[8] V. Chvátal and P. Erdős, Biased positional games, Annals of Discrete Math. 2 (1978), 221-228.

[9] P. Erdős and A. Rényi, On the strength of connectedness of a random graph, Acta Math. Acad. Sci. Hungar. 12 (1961), 261-267. 
[10] P. Erdős and J. Selfridge, On a combinatorial game, J. Comb. Th. Ser. A 14 (1973), 298-301.

[11] H. Gebauer and T. Szabó, Asymptotic random graph intuition and the biased connectivity game, submitted.

[12] D. Hefetz, M. Krivelevich, M. Stojaković and T. Szabó, Avoider-Enforcer: the rules of the game, submitted.

[13] D. Hefetz, M. Krivelevich and T. Szabó, Avoider-Enforcer games, J. Comb. Th. Ser. A 114 (2007), 840-853.

[14] D. Hefetz, M. Krivelevich and T. Szabó, Hamilton cycles in highly connected and expanding graphs, Combinatorica, to appear.

[15] J. Komlós and E. Szemerédi, Limit distributions for the existence of Hamilton circuits in a random graph, Discrete Math. 43 (1983), 55-63. 